\title{
Two component relativistic acceleration and polarized radiation of the parsec-scale AGN jet
}

\author{
Oliver Porth \\ Max Planck Institut für Astronomie \\ 69117 Heidelberg, Germany \\ email: porth@mpia.de
}

\begin{abstract}
We perform axisymmetric simulations of two-component jet acceleration using the special relativistic MHD code PLUTO (Mignone et al., 2007). The inner, thermally driven component constitutes a dilute relativistic plasma originating in a high enthalpy central corona. The second component is a Poynting-dominated wind driven by a global current system. Once a nearstationary state is reached, we solve the polarized Synchrotron radiation transport incorporating self-absorption and (internal) Faraday rotation. With this approach we obtain high-resolution radio maps and spectra that can help in the interpretation of observational data from nearby active galactic nuclei by predicting spine-sheath polarization structures and Faraday rotation gradients.
\end{abstract}

Keywords. galaxies: active - galaxies: jets - ISM: jets and outflows - plasmas - polarization radiation mechanisms: non-thermal - Radiative transfer - relativity

\section{Introduction}

Observations of core-dominated active galactic nuclei (AGN) hold the Synchrotron process responsible for the radio emission. The observed high linear polarization degrees (up to $\sim 30 \%$, Marscher et al., 2002) indicate that the emitting region is characterized by ordered magnetic fields. In current magneto hydrodynamical (MHD) models of jet formation, the magnetic fields form a large-scale helix twisted by the underlying accretion disk or rotating black hole (McKinney, 2006). The bulk of the energy is first carried in terms of Poynting-flux which is gradually converted to kinetic energy by the Lorentz force of the global current system (Vlahakis and Königl, 2004; Komissarov et al., 2007). In order to efficiently transport energy to larger scales and eventually feed a radio lobe, the ordered field structure must survive against instabilities and dissipation to distances well beyond the parsec scale. Under the assumption that the relativistic electrons gyrate around this helical fields while emitting the corresponding Synchrotron radiation, it is interesting to ask how the geometry imprints on the observed radiation (Lyutikov et al. 2005). In the presence of relativistic motion, a kinematic jet model is needed to properly account for the transformation effects such as aberration, polarization swing (Blandford and König, 1979) and relativistic Faraday rotation (Broderick and Loeb, 2009).

In order to provide a global model of the outflow, we simulate the jet comprised of two components: a thermal spine (e.g. Sauty et al. 2004 and N. Globus in this volume) and an outer disk wind similar to Porth and Fendt (2010). Beyond the acceleration region, Meliani and Keppens (2009) showed that the interaction between the two components can give rise to a Raleigh-Taylor-type instability and ultimately cause jet disruption. 


\section{Jet acceleration}

The slow conversion of Poynting-flux to kinetic energy asks for enormous spatial scales that are a challenge to dynamical codes. With a stretched grid, we are able to simulate several thousand gravitational radii and still avoid any causal interaction with the outgoing boundaries. The resulting collimated flow is thus a consequence of jet self-collimation alone. Simulations presented here cover $2555^{2}$ grid cells in the $(r, z)$-plane $\left(12000^{2}\right.$ gravitational radii, $\left.r_{g}\right)$ of which only $1417 \times 2356\left(1200 \times 6000 r_{g}\right)$ are used for the subsequent analysis.

As initial setup we assign a non-rotating corona threaded by a force-free poloidal field with $B_{z}(z=0) \propto r^{-1}$. The pressure gradient is balanced by a point-mass gravity situated $2 r_{g}$ below the simulation domain. We assign boundary conditions for pressure, density and the current distribution and inject a slow-magnetosonic wind into the simulation domain. All remaining variables are treated as outflowing to properly account for the outgoing characteristics of the sub-Alfénic flow.

Specifically, the profiles read

$$
\begin{aligned}
\rho(R) & =\rho_{1}\left[(1-\theta) R+\theta R^{-1.5}\right] \\
p(R) & =p_{1}\left[(1-\theta)\left(1-\rho_{1} \ln R\right)+\theta R^{-2.5}\right] \\
B_{\phi}(r) & =B_{\phi, 1}\left[(1-\theta) r+\theta r^{-s}\right] \\
v_{p}(r) & =v_{s}(r)
\end{aligned}
$$

with $\dagger$

$$
\theta=\left\{\begin{array}{ll}
0 & ; \quad r<1 \\
1 & ; \quad r \geqslant 1
\end{array} .\right.
$$

To treat the hot spine together with the disk-wind, we choose an equation of state satisfying the Taub1948 inequality discussed by Mignone et al., 2005.

Figure 1 illustrates the near-stationary solution and acceleration of the inner and outer component. Helical magnetic fields are shown in the three dimensional rendering of the axisymmetric model. In the right-hand panels energy-conversion along selected field lines is plotted against cylindrical radius, clearly indicating the thermal driving in the spine and magnetic acceleration in the outer component. The total energy flux is only approximately conserved due to more efficient cooling in the central part of the inner component and absence of stationarity in the outer part of the solution. When the jet reaches the end of our simulation box, the outer component is still dominated by Poynting flux. We can see how the acceleration is coupled to the collimation: As the kinetic energy along a field line increases approximately linear with the cylindrical radius, enormous vertical scales are needed in order to follow the complete acceleration process for near-collimated flows (see also the contribution of A. Ferrari in this volume).

\section{Radiation transport}

Before the radiation transport can be conducted, we have to devise physical scales for the simulations. We do so by assuming a radial scale of $6 r_{g}=6 G M_{\bullet} / c^{2}$ for the transition from the inner corona to the Poynting driven jet and by normalizing the energy flow to $10^{44} \mathrm{erg} / \mathrm{s}$. The mock-observations of the parsec-scale AGN core assume a black-hole mass

$\dagger$ where $R$ is the spherical radius to the origin below the simulation domain and $r$ the cylindrical radius. 

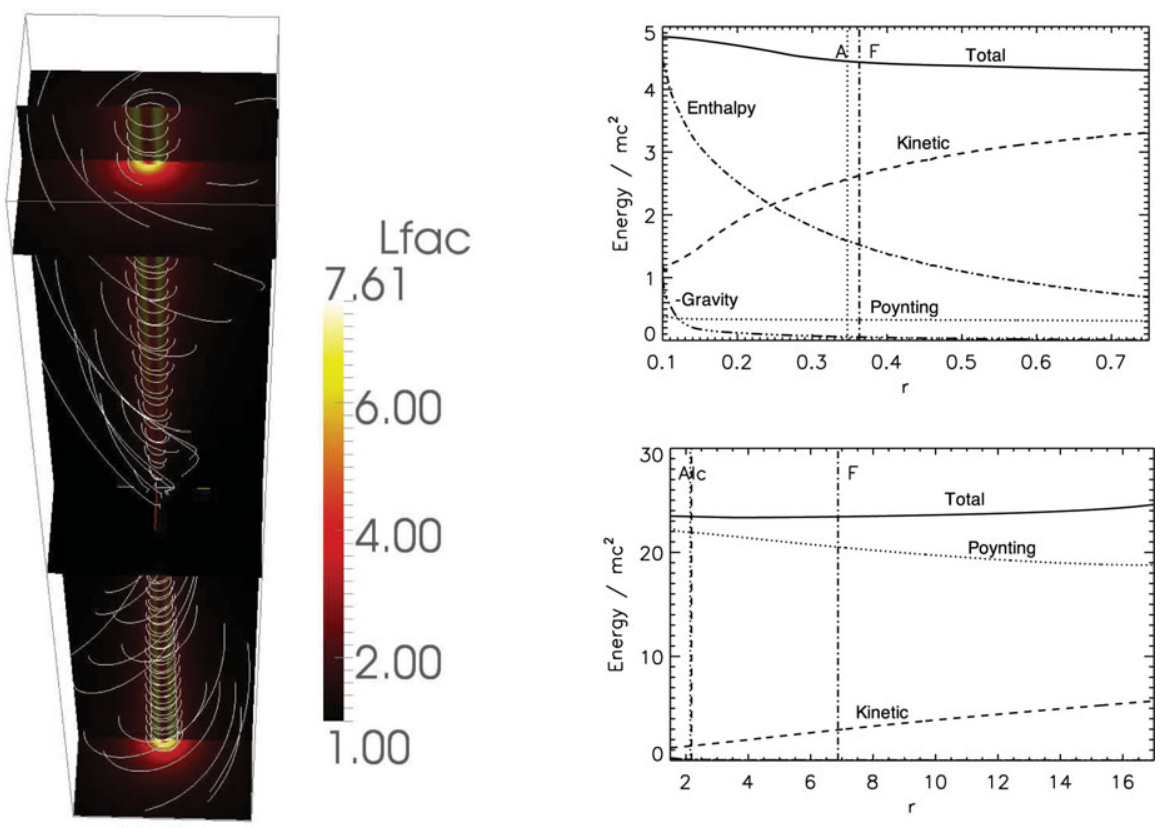

Figure 1. 3D rendering of jet model with asymptotically closed current, $s=1.25$ (left). The slices indicate bulk-flow Lorentz factor and the helical magnetic field lines are traced by the white lines. Right: Acceleration along selected field-lines against the cylindrical radius $\mathrm{r}$ showing thermal acceleration for a field line in the spine (footpoint $r_{\mathrm{fp}}=0.1$, above) and magnetic acceleration in the jet $\left(r_{\mathrm{fp}}=1.5\right.$, below). Vertical lines indicate the crossing of the Alfvèn (A) and fast (F) critical point as well as the light cylinder (lc).

of $M_{\bullet}=10^{9} M_{\odot}$ and a photometric distance of $100 \mathrm{Mpc}$. In this scaling, the active region of the radiation transport covers a physical volume of $0.12 \times 0.12 \times 0.6 \mathrm{pc}^{3}$.

Ray-tracing linearly polarized radiation in the observers system, we solve the corresponding coupled linear equations

$$
\frac{d \mathbf{I}}{d s}=\mathcal{E}-\underline{\mathbf{A}} \mathbf{I}
$$

taking into account relativistic beaming, boosting and swing of the polarization for the three Stokes parameters $\mathbf{I}=\left\{I^{(l)}, I^{(r)}, U^{(l r)}\right\}$. The comoving coefficients of the transfer equation are valid for power-law electron distributions $d n_{e} / d E_{e}=N_{0} E_{e}^{-2 \alpha-1}$ as defined by Pacholczyk (1970). In the following we adopt $\alpha=0.5$ and the lower (upper) cutoff $\gamma_{1}=100\left(\gamma_{\mathrm{u}}=\infty\right)$ to reproduce the empirical SED in the optically thin regime. Faraday rotation is calculated in accordance with Broderick \& Loeb (2009) by utilizing the relativistic generalization for the rotation angle

$$
\frac{d \chi_{F}}{d s}=\frac{e^{3}}{2 \pi m_{e}^{2} c^{2}} \frac{f\left(\gamma_{t}\right) n_{e} D^{2}}{\nu^{2}}(\hat{\mathbf{n}}-\beta) \cdot \mathbf{b}
$$

in relation (3.1) allowing to resolve internal Faraday rotation $\dagger$.

The largest uncertainties concerning the jet radiation in pure MHD models arise due to the lack of information about the location and mechanism of the particle acceleration

$\dagger$ here, $e, m_{e}, n_{e}$ are the comoving electron -charge, -mass and -density, $\mathbf{b}$ is the comoving magnetic field vector and $\hat{\mathbf{n}}$ the (observer-frame) photon direction. The function $f\left(\gamma_{e}\right)$ interpolates between the limits for cold and relativistic electron temperatures $\gamma_{e}$ according to Shcherbakov (2008). 

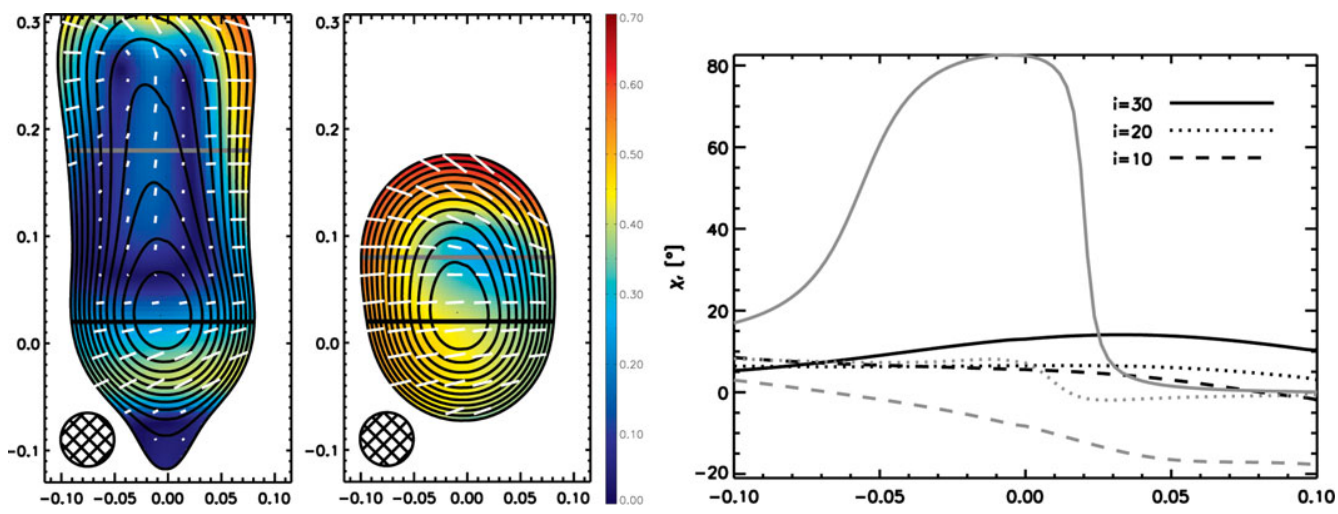

Figure 2. Polarization ê vectors (white sticks) for $i \in\left\{30^{\circ}, 10^{\circ}\right\}$ emitted from regions with co-moving pinches $B_{\phi}^{\prime} / B_{p}^{\prime}>1$. The polarization degree $\Pi_{43 \mathrm{GHz}}$ is indicated by the filling and $I_{43 \mathrm{GHz}}$ contour lines are overplotted in solid black. The latter are spaced by a factor of 2 out to $\simeq 5 \cdot 10^{-4} I_{\nu \text {, peak }}$ where the image is cropped. Spatial scale is given in milli arcseconds and a restoring beam with FWHM=0.05 mas was used. The right-hand panel shows polarization angles for cuts along core (black) and jet (gray).

process. Here we have to resort to a simple recipe by assuming equipartition between the relativistic electrons and the large-scale magnetic field. This treatment is consistent with minimum-energy arguments inversely used to derive magnetic field-strength and electron density from the synchrotron emission Burbidge 1956.

Even in global VLBI experiments, the AGN core emission is almost certainly underresolved and hence we need to investigate the beam-averaging effect on the observables. Figure 2 shows a mock polarization observation at $\nu=43 \mathrm{GHz}$ for two inclinations $i$ (where $i=0^{\circ}$ would directly look into the jet) with a certainly optimistic beam-width of 0.05 mas. To demonstrate how the kinematic information of the jet model imprints on the polarization, Faraday rotation is neglected in this case. For moderate inclinations $i \geqslant 30^{\circ}$ we observe a clear spine and sheath polarization structure tracing the lower pitch of the outer field lines. The polarization degree increases towards the edges and becomes beam-depolarized between perpendicular regions. When approaching the blazar case, polarizations become predominantly perpendicular to the jet direction.

\section{Conclusions}

Using large-scale axisymmetric RMHD simulations combined with rigorous ray-tracing, we demonstrate a way to model AGN core radio emission. Within the simulated domain extending into the parsec scale, our high-energy models accelerate from $\Gamma \simeq 1.1$ to $\Gamma \lesssim 8$ with acceleration still ongoing when the jet leaves the domain.

Depending on the position of the emitting region, the polarization shows characteristic swings by $\sim 90^{\circ}$ that can appear as often observed "spine and sheath" or "jet and core" shift. Although numerous features can be reproduced, the helical field-models always have electric vectors perpendicular to the jet direction when seen "into" the jet and can thus not predict the majority of BL-Lac sources (Marscher et al. 2002).

An investigation that takes into account various particle acceleration recipes, resolution effects and Faraday rotation is in preparation by Porth \& Fendt (2010b). 


\section{Acknowledgements}

This work was carried out under the HPC-EUROPA project (RII3-CT-2003-506079), with the support of the European Community - Research Infrastructure Action under the FP6 "Structuring the European Research Area" Program.

\section{References}

Blandford, R. D. \& Königl, A.: 1979, ApJ 232, 34

Broderick, A. E. \& Loeb, A.: 2009, APjL 703, L104

Burbidge, G. R.: 1956, ApJ 124, 416

Hawley, J. F. \& Krolik, J. H.: 2006, ApJ 641, 103

Komissarov, S. S., Barkov, M. V., Vlahakis, N., \& Königl, A.: 2007, MNRAS 380, 51

Lyutikov, M., Pariev, V. I., \& Gabuzda, D. C.: 2005, MNRAS 360, 869

Marscher, A. P., Jorstad, S. G., Mattox, J. R., \& Wehrle, A. E.: 2002, ApJ 577, 85

McKinney, J. C.: 2006, MNRAS 368, 1561

Meliani, Z. \& Keppens, R.: 2009, ApJ 705, 1594

Mignone, A., Bodo, G., Massaglia, S., Matsakos, T., Tesileanu, O., Zanni, C., \& Ferrari, A.: 2007, Apjs 170, 228

Mignone, A., Plewa, T., \& Bodo, G.: 2005, Apjs 160, 199

Porth, O. \& Fendt, C.: 2010, ApJ 709, 1100

Sauty, C., Meliani, Z., Trussoni, E., \& Tsinganos, K.: 2004, Apss 293, 75

Shcherbakov, R. V.: 2008, ApJ 688, 695

Taub, A. H.: 1948, Physical Review 74, 328

Vlahakis, N. \& Königl, A.: 2004, ApJ 605, 656 